\title{
ASO, ASK and ADNase-B Values in Rheumatic Fever, Rheumatic \\ Heart Disease and Other Infections by \\ Hemolytic Streptococcus
}

\author{
Nobuo Watanabe, Akira Arimura, \\ Munemitsu Kobayashi, Masahiro Oshima
}

$\mathbf{T}$

YPE A hemolytic streptococcus has received attention as the etiologic agent for many diseases such as rheumatic fever, acute glomerulonephritis, scarlet fever, tonsillitis and anaphylactoid purpura (Schönlein-Henoch vasculitis). In addition to ASO measurement, the most widely used serological method for the diagnosis of this disease, measurement of antistreptokinase (ASK) and anti-streptohyaluronidase (ASH) has also been started, and anti-deoxyribonuclease-B (ADNase-B) has also come into use recently. In general, measurement of ASO uncovers about $70-80 \%$ of infections by streptococcus hemolyticus, and addition of ASK and ASH is said to provide positive reaction in $90 \%$ of the patients. According to Wannamaker, 1 ASO occasionally fails to rise during infection by group A streptococcus hemolyticus but ADNase-B is usually elevated, and the titer of the latter is extremely high especially in rheumatic fever.

ASO, ASK and ADNase-B were measured in 40 serum samples from patients with rheumatic fever, rheumatic heart disease, acute glomerulonephritis, anaphylactoid purpura and tonsillitis to study the mutual interrelationship, as reported below.

\section{MATERIALS AND METHODS}

ASO, ASK and ADNase-B were measured in 40 serum samples from 33 patients, 9 with rheumatic fever ( 4 patients in the active stage and 5

Key Words:

Streptococcal infection

Relation between ADNase-B and ASO

Relation between ADNase-B and ASK in the inactive stage), 6 with valvular heart disease, 5 with acute glomerulonephritis, 4 with anaphylactoid purpura and 9 with tonsillitis. ASO was measured by Rantz-Randall method, ASK was measured by Kinase test and ADNase-B was measered by using the Kit for streptonase-B test commercially available from Wampole Laboratories, Dist. The details of these methods were described in reference?

\section{RESULTS}

Among the 40 samples, those obtained during the inactive stage and recovery stage were included. The rate of ADNase-B positive reaction with titers above 240 times was $70 \%$, and that with titers above 340 times, $57.5 \%$. The rate of positive reaction was $50 \%$ for ASO and $45 \%$ for ASK. As to the correlation between ADNase-B and ASO or ASK, the rate of agreement was $65 \%$ for both ASO and ASK, when titers above 240 times of ADNase were defined as positive, and $77.5 \%$ when those above 340 times were defined as positive, also with identical values (Table I, II).

Among 6 patients with ASO $<250 \times$ and ADNase-B $<340 \times, 1$ patient had mitral insufficiency, 4 patients inactive rheumatic fever and 1 patient anaphylactoid purpura. One patient with anaphylactoid purpura and 2 patients with tonsillitis gave the result of ASO $>333 \times$ and ADNase B $<240 x$.

Seven patients with ASK $<1280 \times$, ADNaseB $>340 \times$ included 2 with inactive rheumatic fever, 2 with acute glomerulonephritis and 3 with tonsillitis.

Department of Pediatrics, Kyorin University School of Medicine 
TABLE I RELATION BETWEEN ADNase-B AND ASO (40 CASES)

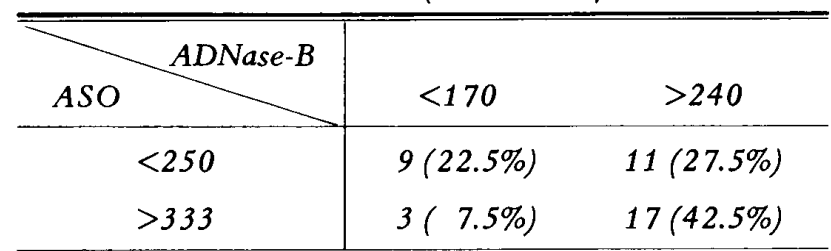

Coincidence Rate $65.0 \%$

\begin{tabular}{|c|c|c|}
\hline${ }_{A S O} A D$ Nase- $B$ & $<240$ & $>340$ \\
\hline$<250$ & $14(35.0 \%)$ & $6(15.0 \%)$ \\
\hline$>333$ & $3(7.5 \%)$ & $17(42.5 \%)$ \\
\hline
\end{tabular}

Coincidence Rate $77.5 \%$

TABLE II RELATION BETWEEN ADNase-B AND ASK (40 CASES)

\begin{tabular}{|c|c|c|}
\hline${ }_{A S K}$ ADNase-B & $<170$ & $>240$ \\
\hline$<1280$ & $10(25.0 \%)$ & $12(30.0 \%)$ \\
\hline$>2560$ & $2(5.0 \%)$ & $16(40.0 \%)$ \\
\hline
\end{tabular}

Coincidence Rate $65.0 \%$

\begin{tabular}{|c|c|c|}
\hline$A S K=A D N$ & $<240$ & $>340$ \\
\hline$<1280$ & $15(37.5 \%)$ & $7(17.5 \%)$ \\
\hline$>2560$ & $2(5.0 \%)$ & $16(40.0 \%)$ \\
\hline
\end{tabular}

Coincidence Rate $77.5 \%$

\section{DISCUSSION}

In patients with infection by hemolytic streptococcus, simultaneous serological tests such as ASO, ASK and ADNase-B do not always give a high value in each. The following explanations have been offered: 1) variable production of exotoxin in different strains, 2) variable intervals required for the normalization of serum titer in different reactions and 3) longer time required for normalization as the titers of the reaction are higher.

According to Stollerman et al ${ }^{3}$, ASO was positive in $78 \%$ of patients with rheumatic fever. Positive reaction was obtained in $90 \%$ by the use of ASO and ASH, and in 95\% by the use of ASO, ASK and ASH. Ayoub et al4 reported the rise of ADNase-B and longer duration of the elevated value of this reaction than other reaction in chorea. According to the report of Hugh et als, rise of ASO was seen in $51 \%$ and that of ADNase-B in $91 \%$ of 101 patients with nephritis which occurred following skin infection by streptococus hemolyticus. High titers of ADNase-B were sustained longer than ASO according to these workers.

The normal value of ADNase-B was below 60 times in young children, 170 times in school children and 85 times in adults according to the results of Klein et al6 In Japan, they are under investigation but appear somewhat higher, so that titers above 240 and 340 times were tentatively considered to be abnormal. The determination of normal range of serum reaction maintained at a high level for a long period is quite difficult as to the selection of "normal" material leaving a room for further study.

In this report, $A D N a s e-B$ was frequently positive and other serum reactions were negative in patients with discordant results. Disagreement between these results on simultaneous determination in the acute stage was experienced in tonsillitis, acute glomerulonephritis and anaphylactoid purpura. Such disagreement was also seen in patients with rheumatic fever after the course of several months to one year. In valvular heart disease, ADNase may stay positive at certain stage even if ASO and ASK were already negative. Such result indicates the history of rheumatic fever in a retrospective way, presenting an important method for the elucidation of the etiology and control of the disease.

\section{REFERENCES}

1. WANNAMAKER LW: The paradox of the antibody response to streptodornase. The usefullness of anti-deoxyribonuclease $B$ as an indication of streptococcal infection in patients with acute rheumatic fever. $A m J$ Med 27: 567, 1959

2. WATANABE N, YAMAMOTO I, OSHIMA M: Antistreptococcal DNAase-B in streptococcal infections. Pediat of Japan 2: 105, 1979

3. STOLLERMAN GH, LEWIS AJ, SCHULTZ I, TARANTA A: Relationship of immune response to group A streptococci to the course of acute, chronic and recurrent rheumatic fever. $A m \mathrm{~J} \mathrm{Med}$ 20: 163,1956

4. AYOUB EM, WANNAMAKER LW: Streptococcal antibody titers in Sydenham's chorea. Pediatrics 38: 946,1966

5. HUGH CD, MARY SAR: Streptococcal immune responses in nephritis after skin infection. $A m J$ Med 56: 333, 1974

6. KLEIN GC, BAKER CN, JONES WL: Upper limits of normal antistreptolysin $\mathrm{O}$ and antideoxyribonuclease B titers. Appl Microbiol 21: 999, 1977 